ORIGINAL

\title{
Flavonoides como agentes quimiopreventivos y terapéuticos contra el cáncer de pulmón
}

\author{
Albert Cabrera ${ }^{a, *}$ y Núria Mach ${ }^{a, b}$ \\ ${ }^{a}$ Àrea de Ciències de la Salut, Institut Internacional de Postgrau de la Universitat Oberta de Catalunya (UOC), Barcelona, \\ España \\ ' INRA, Département de Génétique Animale et Biologie Intégrative, Jouy-en-Josas, France
}

Recibido el 31 de octubre de 2011; aceptado el 30 de octubre de 2012

\section{PALABRAS CLAVE \\ Flavonoides; \\ Cáncer de pulmón; \\ Quimioprevención}

\begin{abstract}
Resumen
El objetivo de la presente revisión es estudiar la relación entre los flavonoides y el cáncer de pulmón, postulando que su consumo habitual en la dieta occidental podría resultar beneficioso para proteger a los pacientes contra el cáncer de pulmón. Se realizó una extensa búsqueda de publicaciones científicas recientes en las siguientes bases de datos electrónicas especializadas: PubMed central (PMC)-NCBI, Elsevier Journal, SciELO España, Scirus y Science Direct, incluyendo estudios en células, animales y humanos sobre el efecto de los flavonoides en la prevención y el desarrollo de cáncer de pulmón. Pese a que los estudios in vitro y en modelos animales muestran la capacidad potencial de los flavonoides para actuar contra diferentes tipos de cáncer, y en especial contra el riesgo de padecer cáncer de pulmón, la presencia de datos contradictorios en los estudios epidemiológicos y la falta de estudios clínicos en humanos actualmente impiden realizar recomendaciones dietéticas basadas en la evidencia científica para los pacientes con cáncer de pulmón. Así, los autores del presente artículo recomiendan seguir los consejos de las guías de alimentación saludable, las cuales promocionan el consumo de alimentos ricos en flavonoides y reflejan el conocimiento necesario para tratar a los pacientes con cáncer de pulmón.

(c) 2012 Asociación Española de Dietistas-Nutricionistas. Publicado por Elsevier España, S.L. Todos los derechos reservados.
\end{abstract}

*Autor para correspondencia

Correo electrónico: mvernet@cst.cat (M. Vernet Vernet). 


\section{KEYWORDS}

Flavonoids;

Lung cancer,

Chemoprevention

\section{Flavonoids as Chemopreventive and Therapeutic Agents Against Lung Cancer}

\begin{abstract}
The objective of the present review is to study the relationship between flavonoids and lung cancer, proposing that their regular consumption in Western diets could be beneficial for protecting patients against lung cancer.

An extensive search of the scientific literature was performed in the following electronic specialized databases (PubMed central (PMC)-NBCl, Elsevier Journal, SciELO Spain, Scirus, Science Direct), including studies in animals, cells, and humans, in order to establish the effect of flavonoids in the prevention and development of lung cancer. Although in vitro and animal studies show the potential ability of flavonoids to act against different types of cancers, especially against lung cancers, the diverse results reported within epidemiological studies, together with the lack of experiments in humans, are the major factors in limiting making dietary recommendations based on scientific evidence for the management of patients with lung cancer. Therefore, the authors of the present study recommend following the dietary health practice guidelines which promotes the consumption of food enriched in flavonoids and reflects the current state of knowledge of an effective and appropriate diet in lung cancer patients.

(c) 2012 Asociación Española de Dietistas-Nutricionistas. Published by Elsevier España, S.L. All rights reserved.
\end{abstract}

\section{Introducción}

La dieta mediterránea se caracteriza por el gran consumo de frutas y hortalizas, cereales integrales, legumbres, aceites vegetales -especialmente de oliva- y frutos secos, el consumo moderado de huevos, pescados y carnes magras y el bajo consumo de carnes rojas y alimentos superfluos ${ }^{1}$. Ya en la década de los cincuenta, se observó que los habitantes de los países mediterráneos tenían una incidencia de cardiopatías mucho menor que los de otros países, lo que demuestra la significativa función de la dieta en la prevención de enfermedades ${ }^{2}$. A partir de los años ochenta, se empezaron a publicar resultados que relacionaban la incidencia de ciertos tipos de cáncer con la dieta, y creció el interés por la alimentación y la nutrición en la prevención de la carcinogénesis ${ }^{3}$. Así, diversos estudios epidemiológicos han identificado relación inversa entre el consumo de frutas y hortalizas ${ }^{4-6}$, legumbres ${ }^{7,8}$ y té 9 algunos tipos de cáncer. Se ha atribuido los efectos beneficiosos de frutas y hortalizas al alto contenido en diferentes compuestos bioactivos $^{10}$, entre ellos los fitoquímicos. El tipo y concentración de fitoquímicos en las células vegetales depende de la variedad, la época del año, el grado de madurez, las condiciones de la cosecha y almacenamiento y el procesamiento ${ }^{2}$. Entre la gran diversidad química de los fitoquímicos, los compuestos fenólicos - como ácidos fenólicos, flavonoides, taninos, estilbenos, curcuminoides, cumarinas, lignanos y quinonas, entre otros - han atraído un interés considerable a causa de su amplia bioactividad, y se han mostrado importantes en la prevención de diferentes tipos de cáncer mediante estudios epidemiológicos y clínicos ${ }^{11,12}$. Dentro de los compuestos fenólicos, los flavonoides son un amplio grupo de moléculas que aparecen de manera natural en las plantas, a las que se atribuyen multitud de efectos terapéuticos ${ }^{13,14}$, incluidas propiedades anticancerígenas, por lo que muchos ensayos se han centrado en el estudio de la relación entre estas biomoléculas, en especial la quercetina ${ }^{15,16}$, con el riesgo de padecer diferentes tipos de cáncer $^{17-19}$, incluido el cáncer de pulmón ${ }^{20-23}$. Algunos metaanálisis y revisiones sistemáticas concluyen que hay relación entre el consumo de flavonoides y el riesgo de cáncer de pulmón en algunos subgrupos de población ${ }^{20,24}$. En este sentido, los estudios observacionales muestran resultados contradictorios sobre la relación entre ingesta de flavonoides y riesgo de padecer cáncer de pulmón dependiendo del subgrupo de población estudiado 22,23,25-27.

El cáncer de pulmón sigue siendo uno de los que causa más muertes en países desarrollados, tanto de varones como de mujeres ${ }^{28,29}$. Existen diferentes hipótesis de mecanismos biológicos plausibles, algunos de ellos aún no verificados mediante evidencia científica en humanos, lo que crea cierto grado de incertidumbre entre el público. Los autores de esta revisión creen oportuno presentar el estado del conocimiento actual entorno a: a) los posibles mecanismos biológicos que podrían estar implicados en el papel de los flavonoides en la prevención del cáncer de pulmón, y b) los resultados de los principales estudios que relacionan el consumo de flavonoides con el riesgo de padecer cáncer de pulmón.

\section{Flavonoides}

Los flavonoides son moléculas ampliamente distribuidas dentro del reino vegetal y se consumen habitualmente en frutas, hortalizas, legumbres, cereales integrales y bebidas como el té ${ }^{30}$. Constituyen un grupo de más de 6.000 moléculas que forman parte de diversos procesos bioquímicos y fi- 
siológicos en las plantas y su relación con el entorno ${ }^{31}$. Dentro de este amplio grupo de moléculas, las hay pigmentadas como las antocianinas, que dan su color característico a hojas, flores y frutos de ciertas plantas, y otras no pigmentadas. Están implicadas en un gran número de procesos como, por ejemplo, la atracción de agentes polinizantes mediante la coloración de órganos florales ${ }^{32}$, la germinación del tubo polínico ${ }^{33}$, la defensa contra los insectos actuando como insecticidas ${ }^{34}$ o como agente antifúngico ${ }^{35}$.

\section{Estructura de los flavonoides}

El término flavonoide describe un amplio grupo de moléculas que están constituidas por la base $C_{6}-C_{3}-C_{6} 0$, más específicamente, la funcionalidad fenilbenzopirano. Dependiendo de dónde se une el grupo fenil a la fracción benzopirano, podemos dividir este grupo de productos naturales en: flavonoides (2-fenilbenzopiranos), isoflavonoides (3-fenilbenzopiranos) y neoflavonoides (4-fenilbenzopiranos) ${ }^{36}$ (fig. 1). Dentro del grupo, también se incluyen otras moléculas como las chalconas, hidrochalconas, auronas y auronoles.

Los flavonoides, isoflavonoides y neoflavonoides a su vez se dividen en diferentes grupos dependiendo por ejemplo del grado de oxidación y saturación del heterociclo central, o por la presencia de heterociclos adicionales a la estructura base ${ }^{36}$ (fig. 2). Estos compuestos se encuentran sólo en plantas, principalmente en forma de glucósidos, y pueden encontrarse comúnmente en hojas, flores, tejidos y partes leñosas como el tallo o la corteza ${ }^{37}$.

\section{Los flavonoides en la dieta}

A causa de su presencia común en el reino vegetal, los flavonoides forman parte de la dieta animal y humana ${ }^{36}$. Debido a la importancia que los flavonoides están adquiriendo, el Departamento de Agricultura de Estados Unidos está llevando a cabo un estudio sobre las cantidades de estos compuestos que los alimentos contienen ${ }^{38}$. La tabla 1 describe los contenidos en flavonoides mayoritarios de diferentes alimentos (entre otros, cebollas, manzanas y té). Asimismo, los flavonoides también se encuentran en plantas usadas tradicionalmente como medicinales ${ }^{37}$.

\section{Los flavonoides como compuestos bioactivos}

Los flavonoides tienen la capacidad de afectar a diversos sistemas enzimáticos y funciones biológicas de los mamíferos $^{39}$. La capacidad antioxidante es la más estudiada entre sus funciones biológicas. No obstante, muchos flavonoides también han mostrado actividad antitrombótica, inhibición de la activación leucocitaria o incluso actividad vasodilatadora, con lo que tienen un papel potencial contra enfermedades cardiovasculares ${ }^{40}$. Otros flavonoides, derivados de plantas usadas tradicionalmente como medicinales, han mostrado actividad antibacteriana, antifúngica y antiviral (especialmente las isoflavonas) contra especies resistentes a antibióticos ${ }^{41,42}$ y contra infecciones por la bacteria Helicobacter pylori ${ }^{43}$. Por último, también se les atribuyen posibles propiedades antialérgicas ${ }^{44}$ y pro-<smiles>c1ccc(C2CCc3ccccc3O2)cc1</smiles>

Flavonoides<smiles>c1ccc(C2COc3ccccc3C2)cc1</smiles>

Isoflavonoides<smiles>c1ccc(C2CCOc3ccccc32)cc1</smiles>

Neoflavonoides<smiles>O=C(/C=C/c1ccccc1)c1ccccc1O</smiles>

2'-OH-chalcona<smiles>O=C(CCc1ccccc1)c1ccccc1O</smiles>

2'-OH-dihidrochalcona<smiles>O=C(/C=C/c1ccccc1O)c1ccccc1</smiles>

2'-OH-retro-chalcona<smiles>O=C1/C(=C\c2ccccc2)Oc2ccccc21</smiles>

Aurona<smiles>O=C1c2ccccc2OC1(O)Cc1ccccc1</smiles>

Auronol

Figura 1 Tipos básicos de flavonoides según su estructura. Adaptado de Grotewold ${ }^{36}$. 
<smiles>c1ccc(C2CCc3ccccc3O2)cc1</smiles>

Flavano<smiles>O=c1c(O)c(-c2ccccc2)oc2ccccc12</smiles>

Flavonol<smiles>OC1CC(c2ccccc2)Oc2ccccc21</smiles>

Flavan-4-ol

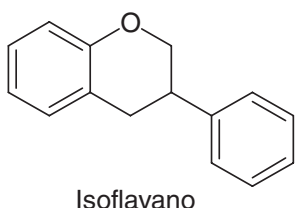<smiles>C1=C(c2ccccc2)COc2ccccc21</smiles>

Isoflav-3-eno<smiles>O=c1oc2ccccc2c2oc3ccccc3c12</smiles>

Cumestano<smiles>O=C1c2ccccc2OC2Oc3ccccc3C12</smiles>

Cumaronacromona<smiles>O=c1cc(-c2ccccc2)c2ccccc2o1</smiles>

4-Arilcumarina<smiles>O=C1CC(c2ccccc2)Oc2ccccc21</smiles>

Flavanona<smiles>O=C1c2ccccc2OC(c2ccccc2)C1O</smiles>

Dihidroflavonol<smiles>OC1c2ccccc2OC(c2ccccc2)C1O</smiles><smiles></smiles>

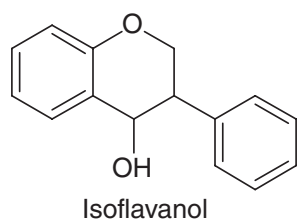<smiles>O=c1oc2ccccc2cc1-c1ccccc1</smiles><smiles>c1ccc2c(c1)OC1c3ccccc3OCC21</smiles>

Pterocarpano<smiles>O=C1CC(c2ccccc2)c2ccccc2O1</smiles>

3,4-Dihidro-4-arilcumarina<smiles>O=c1cc(-c2ccccc2)oc2ccccc12</smiles>

Flavona<smiles>OC1Cc2ccccc2OC1c1ccccc1</smiles>

Flavan-3-ol<smiles>O=C1c2ccccc2OCC1c1ccccc1</smiles><smiles>CCOCCOc1ccccc1C1COc2ccccc2C1</smiles><smiles>C1=C2c3ccccc3OC2Oc2ccccc21</smiles>

Cumaronacromeno<smiles>CC1(C)C=C(c2ccccc2)c2ccccc2O1</smiles>

Neoflaveno

Figura 2 Subclases de flavonoides. Adaptado de Grotewold ${ }^{36}$. 
Tabla 1 Alimentos comunes y su cantidad de flavonoides ${ }^{38}$

\begin{tabular}{|c|c|c|c|c|c|c|c|c|}
\hline Alimento & Quercetina & Kaempferol & Miricetina & Apigenina & Luteolina & Naringenina & Hesperidina & Eriodictiol \\
\hline Alcachofa & - & - & - & 4,70 & 2,27 & - & - & - \\
\hline Alcaparra & 233,84 & 259,19 & - & - & - & - & - & - \\
\hline Apio & - & - & - & 2,34 & 0,63 & - & - & - \\
\hline Brócoli & $2,25-2,51$ & $2,49-4,01$ & 0,01 & - & 0,86 & - & - & - \\
\hline Cacao & 20,13 & - & - & - & - & - & - & - \\
\hline Calabaza & - & - & - & - & 1,63 & - & - & - \\
\hline Cebolla & $12,60-33,43$ & $1,03-4,10$ & $0,03-1,07$ & - & - & - & - & - \\
\hline Cereza & $2,64-3,20$ & - & - & - & - & - & - & - \\
\hline Ciruela & 12,45 & 0,01 & 0,01 & - & - & - & - & - \\
\hline Col rizada & $0,07-7,71$ & $0-26,74$ & - & - & - & - & - & - \\
\hline Espárrago & 12,40 & - & - & - & - & - & - & - \\
\hline Kiwi & - & - & - & 0 & $0-1,12$ & - & - & - \\
\hline Lechuga & $1,42-11,99$ & $0-0,015$ & $0-0,09$ & $0-0,16$ & $0-1,58$ & - & - & - \\
\hline Manzana & $0,43-4,70$ & $0,01-0,02$ & 0,01 & - & - & - & - & - \\
\hline Miel & - & - & - & 0,05 & 0,63 & - & - & - \\
\hline Orégano & $0-7,3$ & - & $0-2,1$ & $0-2,51$ & $1-25,10$ & - & - & - \\
\hline Pera & 4,51 & - & - & - & - & - & - & - \\
\hline Perejil & - & - & - & 225,93 & 1,24 & - & - & - \\
\hline Pimiento & - & - & - & - & $0,61-4,98$ & - & - & - \\
\hline Té negro hojas & 199,75 & 126,66 & 42,24 & - & - & - & - & - \\
\hline Té negro infusión & 1,99 & 1,31 & 0,45 & - & - & - & - & - \\
\hline Té verde hojas & 223,97 & 147,55 & 104,76 & 12,03 & 0,17 & - & - & - \\
\hline Té verde infusión & $0,21-2,69$ & $0,32-1,42$ & $0,58-1,10$ & 0,17 & 0,17 & - & - & - \\
\hline Tomate & $0,03-4,12$ & $0,01-0,1$ & $0,01-0,05$ & - & - & - & - & - \\
\hline Tomillo & - & - & - & 2,50 & 45,26 & - & - & - \\
\hline Uva & $1,38-2,54$ & - & $0,01-0,45$ & - & 1,30 & - & - & - \\
\hline Vino tinto & 2,16 & 0,24 & 0,94 & - & - & 1,77 & 0,63 & - \\
\hline Zumo de limón & - & - & - & - & $0-1,83$ & 1,38 & 14,47 & 4,88 \\
\hline Zumo de naranja & - & - & - & - & - & 2,19 & 11,26 & 0,17 \\
\hline Zumo de pomelo & - & - & - & - & - & $17,9-20,06$ & $0,78-3,42$ & $0-0,65$ \\
\hline Cereza & $3,83-6,97$ & $0-0,05$ & - & - & $0,30-1,31$ & - & - & - \\
\hline Harina de soja & - & - & - & - & - & $67,69-89,46$ & $85,12-98,77$ & $16,12-20,02$ \\
\hline Manzana & $3,79-7,10$ & $0-0,01$ & $0,35-1,52$ & $0,11-0,26$ & $0,62-3,66$ & - & - & - \\
\hline Mora & 4,66 & - & 0,10 & 0,68 & 37,06 & - & - & - \\
\hline Queso de soja & - & - & - & - & - & $0,80-11,14$ & $1,70-4,10$ & $1,50-7,80$ \\
\hline Semilla de soja & - & - & - & - & - & $39,78-89,32$ & $9,01-22,37$ & $27,77-78,86$ \\
\hline Uva & $1,20-8,68$ & $0,17-2,81$ & $0-0,08$ & - & $0,82-10,14$ & - & - & - \\
\hline Vino tinto & 3,28 & 0,01 & 0,06 & - & 7,02 & 0,10 & - & - \\
\hline
\end{tabular}

Cantidades de flavonoides en $\mathrm{mg} / 100 \mathrm{~g}$ de porción comestible que contienen. Los intervalos de valores incluyen el máximo y el mínimo de los flavonoides mayoritarios encontrados en determinado alimento en las bases de datos realizadas por el Departamento de Agricultura de Estados Unidos. En caso de no especificarse la variedad (p. ej., "uva"), se consideran todas las especies posibles. En cuanto a la infusión, todos los valores han sido estandarizados al 1\%, o sea, $1 \mathrm{~g}$ de hojas de té en $100 \mathrm{ml}$ de agua.

tectoras del sistema vascular ${ }^{45}$ y del hígado ${ }^{46}$ y efectos en trastornos oculares ${ }^{47}$ y enfermedades neurodegenerativas $^{48}$. Asimismo, es importante mencionar la posible asociación entre el consumo de flavonoides y la prevención y el desarrollo de algunos tipos de cáncer, especialmente el de pulmón ${ }^{20,24}$.

\section{La carcinogénesis y el cáncer de pulmón}

El cáncer se puede considerar una enfermedad genética causada por la adquisición secuencial de mutaciones en ge- nes implicados en la proliferación y la muerte celulares. El origen de este proceso se encuentra en el daño producido en el ADN celular. El daño celular puede ser causado por: a) procesos endógenos, como pueden ser errores en la replicación del ADN, inestabilidad química intrínseca de algunas bases que forman el ADN, por el ataque de radicales libres generados durante el metabolismo, o b) por procesos exógenos como la radiación ionizante, radiación ultravioleta, carcinógenos químicos, algunos virus y bacterias o la alimentación, entre otros. A pesar de todos estos factores, la probabilidad de que la célula con el ADN dañado se convierta en cancerígena es baja, y existen mecanismos de repara- 


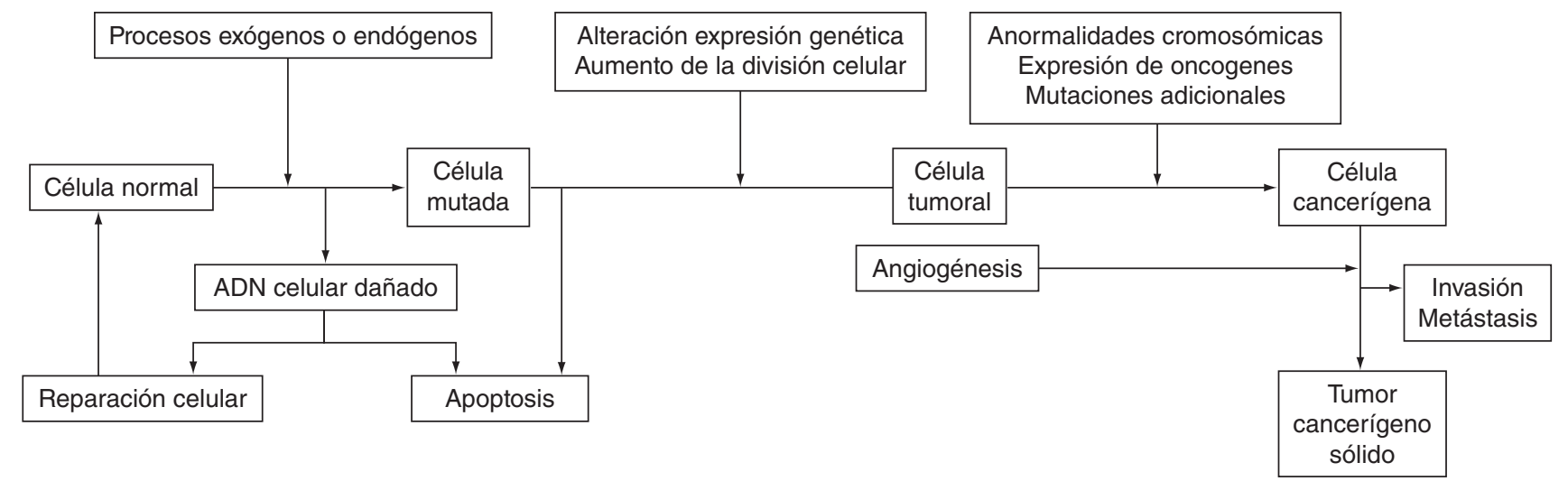

Figura 3 Representación de la transformación de una célula normal en un tumor cancerígeno sólido. Adaptado de Mehta et al ${ }^{10}$.

ción del ADN y de inducción de la muerte celular si la reparación no surtiera efecto (fig. 3).

Los principales genes implicados en las oncopatías son los oncogenes, los genes supresores de tumores y los que afectan a la reparación del ADN. Los protooncogenes controlan el crecimiento y la división celular mediante los factores de crecimiento, que se encuentran ligados a receptores en la superficie celular. Esta unión desencadena la activación de los factores de transcripción (FT) dentro del núcleo celular. La activación de los FT activa la expresión de los genes requeridos para el crecimiento y la proliferación celular. La sobreexpresión de los oncogenes potencia la división y el crecimiento constante de la célula. Los genes supresores de tumores codifican proteínas que afectan también al crecimiento y la división celular, por lo que su alteración en expresión permite a la célula dividirse y crecer incontroladamente. Por último, hay una serie de genes implicados en la reparación del ADN que codifican proteínas que actúan en la corrección de errores durante la replicación del ADN previa a la división celular. Los cambios de expresión de estos genes permiten que se produzcan cambios en los genes implicados en el cáncer definidos anteriormente.

Además de estos cambios en el transcriptoma y el proteoma celular, es necesaria la activación del proceso de angiogénesis, que consiste en el desarrollo de vasos sanguíneos que permiten al tumor obtener los nutrientes necesarios para su crecimiento y eliminar los productos de su metabolismo 49 .

El de pulmón es el tipo de cáncer más común, y tiene mal diagnóstico debido a su tendencia a la invasión local y posterior metástasis. Aproximadamente $2 / 3$ metástasis se producen en zonas alejadas como el cerebro, el pulmón contralateral y el hueso ${ }^{50}$. La tasa de supervivencia en este tipo de cáncer en los 5 años posteriores a su detección es de aproximadamente el $15 \%{ }^{51}$. Un $80-85 \%$ de los casos corresponden a cáncer de pulmón de células no pequeñas, de los cuales el $70 \%$ presenta un estado avanzado de la enfermedad en el momento del diagnóstico. Actualmente se opta en primer lugar por un tratamiento que incluye quimioterapia, aunque algunos pacientes se vuelven resistentes al tratamiento en estadios avanzados de la enfermedad $^{52}$.

\section{Flavonoides y cáncer de pulmón}

Diversos artículos han revisado la posible actividad preventiva del consumo de ciertos alimentos contra el cáncer de pulmón, y señalan a los flavonoides (y especialmente las catequinas) como los compuestos bioactivos que causan dicha actividad (tabla 1). Los flavonoides pueden interferir en la carcinogénesis participando en el bloqueo o la supresión de diversos procesos ${ }^{53}$. Entre los mecanismos biológicos que han recibido más atención debido a su relación con el proceso de carcinogénesis, se cuentan metabolismo del cáncer, reparación del $A D N$, proliferación celular, apoptosis, ciclo celular, angiogénesis y metástasis ${ }^{25}$ (fig. 3).

\section{Análisis mecanístico del potencial de acción de los flavonoides como agentes contra el cáncer de pulmón: estudios en modelos celulares y en modelos animales}

Diversos estudios in vitro e in vivo en modelos animales muestran la capacidad y los mecanismos mediante los cuales los flavonoides podrían interferir en los diversos procesos relacionados con el cáncer de pulmón. En la tabla 2 se presenta un resumen de los principales estudios realizados en modelos celulares y animales al respecto ${ }^{51,52,54-70}$. Un estudio in vivo con ratones a los que se induce cáncer mediante la molécula benzo(a)pireno, presente en el humo del tabaco, muestra la capacidad de la fisetina, un flavonol presente en frutas y hortalizas (fresa, manzana, caqui, uva, cebolla y pepino), para inhibir la proliferación celular y el desarrollo de tumores, gracias a sus propiedades antioxidantes ${ }^{68}$. La inducción del cáncer de pulmón mediante benzo(a)pireno implica, por otra parte, la bioactivación de procarcinógeno mediante unas enzimas codificadas por el gen CYP1A1 (citocromo P450). La quercetina, un flavonol presente en gran número de frutas y verduras (tabla 1) ha mostrado in vitro una acción inhibitoria de esta vía ${ }^{58}$. En un estudio in vitro se observó que el kaempferol, flavonol que podemos encontrar en hortalizas como la col rizada o el brócoli (tabla 1), podría inducir apoptosis en células cancerígenas del pulmón $\mathrm{H} 460$ afectando a las dos vías metabólicas en la apoptosis, las denominadas dependientes e independientes de $\operatorname{caspasa}^{60}$. Las caspasas son un grupo de 
Tabla 2 Recopilación de estudios preclínicos y clínicos sobre flavonoides y derivados contra el cáncer de pulmón

\begin{tabular}{|c|c|c|c|c|}
\hline Moléculas & $\begin{array}{l}\text { Tipo de } \\
\text { estudio }\end{array}$ & Año & Resultado & Mecanismo de acción propuesto \\
\hline Kaempferol & In vitro & 2003 & $\begin{array}{l}\text { Inducción de apoptosis en células } \\
\text { A549 }\end{array}$ & Activación MEK-MAPK ${ }^{54}$ \\
\hline Quercetina & In vitro & 2004 & $\begin{array}{l}\text { Inhibición crecimiento células A549 } \\
\text { e inducción de apoptosis }\end{array}$ & Activación vía señalización MEK-ERK ${ }^{55}$ \\
\hline Quercetina & In vitro & 2004 & $\begin{array}{l}\text { Inhibición del crecimiento celular } \\
\text { e inducción de apoptosis en células } \\
\text { A549 y H1299 mediante tratamiento } \\
\text { con quercetina } 20-80 \mu \mathrm{m} \text { durante } 24 \mathrm{~h}\end{array}$ & Regulación de la vía p53/survivina ${ }^{56}$ \\
\hline $\begin{array}{l}\text { Complejo de } \\
3 \text { aminoflavona y } \\
\text { Cis-Pt(II) }\end{array}$ & In vitro & 2005 & $\begin{array}{l}\text { Inducción de apoptosis en células } \\
\text { A549 }\end{array}$ & Activación del gen p53 y BAX ${ }^{57}$ \\
\hline $\begin{array}{l}\text { Quercetina y } \\
\text { naringenina }\end{array}$ & In vitro & 2005 & $\begin{array}{l}\text { Efectos inhibitorios de la activación } \\
\text { de los mecanismos del cáncer de } \\
\text { pulmón inducidos por benzo(a)pireno, } \\
\text { especiamente la quercetina }\end{array}$ & $\begin{array}{l}\text { Inhibición de la formación de } \\
\text { carcinógenos mediante la vía regulada } \\
\text { por el gen CYP1A1 }\end{array}$ \\
\hline $\begin{array}{l}\text { Flavanona } \\
\text { y 2'OH flavanona }\end{array}$ & In vitro & 2007 & $\begin{array}{l}\text { Inhibición de la invasión celular en } \\
\text { A549 después de un tratamiento de } \\
72 \text { h con } 10 \mu \mathrm{m} \text {. Inhibición de la } \\
\text { metástasis en tratamientos a largo } \\
\text { plazo }\end{array}$ & Disminución expresión MMP-2 y u-PA ${ }^{51}$ \\
\hline & In vivo & & $\begin{array}{l}\text { Inhibición de metástasis en ratas. } \\
\text { Inhibición del crecimiento de nodulos } \\
\text { metastásicos }\end{array}$ & \\
\hline Quercetina & In vitro & 2007 & $\begin{array}{l}\text { Inducción de apoptosis de células } \\
\text { A549, H460 y H1299, sin presentar } \\
\text { toxicidad células epiteliales normales } \\
\text { de los bronquios HCCBE-2 y HCCBE3 }\end{array}$ & $\begin{array}{l}\text { Sensibilizacion ligando TRAIL mediante } \\
\text { ligando DR5 y supresión de la expresión } \\
\text { de la survivina mediada por } \mathrm{Akt}^{59}\end{array}$ \\
\hline Kaempferol & In vitro & 2007 & Inducción de apoptosis en H460 & $\begin{array}{l}\text { Cambio en la expresión de marcadores } \\
\text { de apoptosis como caspas } 3 \text { y AIF. Vía } \\
\text { indirecta mediante especies reactivas } \\
\text { de oxígeno. Expresión aumentada de la } \\
\text { enzima MnSOD }{ }^{60}\end{array}$ \\
\hline Wogonina & In vitro & 2008 & $\begin{array}{l}\text { Inhibición de la inflamación producida } \\
\text { en células A549 }\end{array}$ & $\begin{array}{l}\text { Inhibición de la expresión del gen } \mathrm{COX}-2 \\
\text { inducida mediante } \mathrm{PMA}^{61}\end{array}$ \\
\hline Isoramnetina & $\begin{array}{l}\text { In vitro } \\
\text { In vivo }\end{array}$ & 2008 & $\begin{array}{l}\text { Inducción de apoptosis } \\
\text { Reducción de tamaño y peso de los } \\
\text { tumores inducidos en ratones } \\
\text { mediante isoramentina a partir de } \\
0,5 \mathrm{mg} / \mathrm{kg}\end{array}$ & Mecanismo dependiente de caspasa ${ }^{62}$ \\
\hline Fisetina & In vitro & 2009 & $\begin{array}{l}\text { Efecto antimetastásico en células } \\
\text { A549 }\end{array}$ & $\begin{array}{l}\text { Disminución expresión MMP-2 y u-PA. } \\
\text { Inactivación de la vía de señalización } \\
\text { ERK }^{63}\end{array}$ \\
\hline Baicaleína & $\begin{array}{l}\text { In vitro } \\
\text { In vivo }\end{array}$ & 2010 & $\begin{array}{l}\text { Efectos citotóxicos en células A549 y } \\
\text { poca alteración en fibroblastos. } \\
\text { Supresión de crecimiento del tumor } \\
\text { en ratas y supervivencia prolongada } \\
\text { sin toxicidad sistémica }\end{array}$ & $\begin{array}{l}\text { Aumento de la actividad de SOD e } \\
\text { inhibición de HIF- } 1 \alpha^{64}\end{array}$ \\
\hline Didimina & In vivo & 2010 & $\begin{array}{l}\text { Inhibición crecimiento celular e } \\
\text { inducción de apoptosis en células } \\
\text { A549 y H460 } \\
\text { Supresión de crecimiento del tumor } \\
\text { (A549) en ratas sin toxicidad } \\
\text { sistémica }\end{array}$ & $\begin{array}{l}\text { Aumento de la actividad de los ligandos } \\
\text { Fas y caspasa } 8^{65}\end{array}$ \\
\hline
\end{tabular}


Tabla 2 Recopilación de estudios preclínicos y clínicos sobre flavonoides y derivados contra el cáncer de pulmón (continuación)

\begin{tabular}{|c|c|c|c|c|}
\hline Moléculas & $\begin{array}{l}\text { Tipo de } \\
\text { estudio }\end{array}$ & Año & Resultado & Mecanismo de acción propuesto \\
\hline $\begin{array}{l}20 \text { flavonoides y } \\
\text { análogos }\end{array}$ & In vitro & 2010 & $\begin{array}{l}\text { Disminución del factor VGE en células } \\
\text { H157 que aparece en estadios } \\
\text { avanzados del cáncer de pulmón }\end{array}$ & $\begin{array}{l}\text { Inhibición de fosforilación de STAT3 por } \\
\text { hipoxia }^{66}\end{array}$ \\
\hline \multirow{2}{*}{$\begin{array}{l}\text { Quercetina } \\
\text { combinada con } \\
\text { quimoterapia } \\
\text { tradicional (cisplatino } \\
\text { y gemzitabina) }\end{array}$} & In vitro & 2010 & $\begin{array}{l}\text { Disminución de la supervivencia de } \\
\text { células madre cancerosas de pulmón }\end{array}$ & $\begin{array}{l}\text { Inactivación de la vía P38MAPK- } \\
\text { MAPKAPK2-Hsp2767 }\end{array}$ \\
\hline & In vivo & & Inhibición de la progresión del tumor & \\
\hline Luteolina & In vitro & 2011 & $\begin{array}{l}\text { Detención del ciclo celular y } \\
\text { apoptosis en células A549 }\end{array}$ & $\begin{array}{l}\text { Activación JNK e inhibición de } \\
\text { translocación de NF-KB(P65) }\end{array}$ \\
\hline $\begin{array}{l}\text { ASA404 (análogo } \\
\text { flavonoide) }\end{array}$ & In vivo & 2011 & $\begin{array}{l}\text { Medicamento en fase clínica III para } \\
\text { tratamiento de cáncer de pulmón de } \\
\text { células no pequeñas }\end{array}$ & $\begin{array}{l}\text { Mediante varios mecanismos causa } \\
\text { interrupción en el suministro de sangre } \\
\text { al tumor, lo que causa su muerte }{ }^{69}\end{array}$ \\
\hline Fisetina & In vivo & 2011 & $\begin{array}{l}\text { Inhibición de la proliferación celular } \\
\text { y desarrollo del tumor en cáncer } \\
\text { inducido por benzo(a)pireno }\end{array}$ & Inhibición de la formación de LPO ${ }^{68}$ \\
\hline Naringenina & In vitro & 2011 & $\begin{array}{l}\text { Inducción de apoptosis de células } \\
\text { A549 selectivamente, no afecta a } \\
\text { fibroblastos }\end{array}$ & $\begin{array}{l}\text { Apoptosis inducida en células } \\
\text { cancerígenas mediante el receptor DR5 } \\
\text { en el ligando TRAIL }{ }^{70}\end{array}$ \\
\hline
\end{tabular}

cisteinproteasas (más de 14 en esta familia) involucradas en diversos procesos de muerte celular programada. La quercetina también ha mostrado capacidad inhibitoria del crecimiento celular y capacidad para inducir apoptosis en células A549, mediante la activación de sistemas dependientes de caspasas $^{55}$ (vía MAPK/ERK). De un modo parecido, la isoramnetina de la familia de los flavonoles podría actuar mediante la activación de una cascada de reacciones que forman parte del sistema proapoptótico mitocondria-citocromo C-caspasa 9. Esta actividad observada in vitro se ha confirmado in vivo en modelos animales, y se observa una clara disminución del tamaño y el peso de tumores inducidos en ratones a partir de dosis de $0,5 \mathrm{mg} / \mathrm{kg}^{62}$. Las vías de apoptosis independientes de caspasa están asociadas con el apoptosis-induced factor (AIF), proteína que se libera y tiene la capacidad de inducir una condensación y fragmentación nuclear bajo una pérdida de la integridad de la membrana celular. Otra vía que ha atraído la atención es la llamada tumor necrosis factor-related ligand (TRAIL), concretamente el receptor 5 (DR5 o también TRAIL-R2), que se encuentra en la superficie celular y podría inducir apoptosis preferentemente de células transformadas o malignas. La naringenina, flavanona presente en cítricos, y en especial el pomelo (tabla 1), tiene la capacidad de activar esta vía y producir apoptosis en células tipo A549 sin inhibir el crecimiento de fibroblastos normales tipo WI-3870. De modo parecido, la quercetina podría potenciar la sensibilización de esta vía en células cancerígenas no pequeñas de pulmón tipo A549, H460 y H129959. Paralelamente, la baicaleína, flavonoide presente en la planta Scutellaria baicalensis, ha mostrado capacidad de inducir la sobreexpresión del gen que codifica la enzima supeóxido dismutasa (SOD) en célu- las cancerígenas, pues aumenta la producción de peróxido de hidrógeno, suprime la acumulación de la proteína hipóxica HIF-1 $\alpha$, inhibe selectivamente el crecimiento celular en células cancerígenas de pulmón A549 y muestra mínima alteración en células normales ${ }^{64}$. Por su parte, la luteolina, una flavona presente en buena parte de las especias (tabla 1), podría actuar sobre enzimas clave en las vías de señalización celular, detener el ciclo celular e inducir la apoptosis de células cancerígenas de pulmón A54952. La administración de flavononas a ratones ha mostrado inhibición de metástasis mediante la modificación de las vías de señalización MAPK (mitogen activated protein kinases), implicadas en procesos patológicos como inflamación, adhesión de células cancerígenas, invasión, migración y angiogénesis. Se ha descrito la activación de las MAPK y posteriormente los factores AP-1 (activator protein-1), NF$\kappa \mathrm{B}$ (nuclear factor $\kappa B$ ) y ETS-1 (E-twenty six) por aumentar la expresión de las metaloproteasas, por lo que promueven la invasión tumoral ${ }^{51}$. La fisetina también ha mostrado in vitro capacidad para inhibir la migración e invasión de células cancerígenas de pulmón A549 mediante la inactivación de la vía ERK1/2 (extracelular signal-regulated kinases). Estos resultados se han confirmado en estudios in vivo en modelos animales, con inhibición de metástasis en ratones ${ }^{50}$. Por último, los tumores en estado avanzado muestran gran cantidad de proteína VEGF (vascular endothelial growth factor). Se cree que esta proteína es la principal reguladora de la angiogénesis fisiológica y patológica. El estudio in vitro de los efectos de diversos flavonoides en dicha proteína indica que los derivados de las flavonas tienen el potencial de inhibir la expresión de este factor en células no pequeñas cancerígenas de pulmón tipo $\mathrm{H} 157^{66}$. 
A pesar de que muchos flavonoides hayan mostrado, en modelos celulares y animales, ciertas capacidades anticancerígenas, parece poco probable que una sola sustancia actúe de manera independiente, y atendiendo a la gran variedad de flavonoides presentes en los alimentos y de estructuras y actividades de cada uno de ellos, o incluso que las variaciones en el anillo central de los flavonoides y el número, el tipo y la posición de los sustituyentes podría modificar su actividad anticancerígena ${ }^{52,71}$, es más probable que estos actúen en sinergia. Asimismo, cabe destacar que los hallazgos realizados en este tipo de estudios, si bien sirven para ofrecer hipótesis científicas y mecanismos mediante los cuales los flavonoides podrían desarrollar una acción protectora contra el cáncer de pulmón, no se puede trasladar dichos hallazgos al ser humano ni se puede establecer una relación causal, sino que se requieren muchos estudios clínicos realizados en humanos.

\section{Relación entre la ingesta de flavonoides y la prevención del cáncer de pulmón: estudios observacionales en humanos}

El análisis de las conclusiones de los estudios observacionales identifica posibles factores que permitirían disminuir el riesgo de padecer ciertas enfermedades o incluso prevenirlas.

Un estudio publicado en 2001, realizado en una cohorte de 27.110 varones fumadores de mediana edad con un seguimiento de más de 6 años, concluye que la ingesta de flavonoles y flavonas tiene relación inversa con el riesgo de cáncer de pulmón ${ }^{26}$. Un estudio publicado en 2008, realizado en una cohorte de 34.708 mujeres posmenopáusicas con un seguimiento de 18 años, concluye que hay menos incidencia de cáncer de pulmón entre las mujeres que consumieron más flavonoides (especialmente flavanonas y proantocianidinas), sobre todo en los subgrupos de fumadoras y ex fumadoras ${ }^{72}$. Otro estudio publicado el mismo año y realizado en una cohorte de 2.590 varones fumadores de mediana edad, durante un periodo de seguimiento de más de 16 años, analizó la relación de 26 flavonoides y el riesgo de cáncer de pulmón, y concluyó que quienes consumieron más cantidad de flavonoides totales mostraban menos riesgo relativo de cáncer de pulmón ${ }^{73}$. Paradójicamente, en otro estudio prospectivo publicado en 2010 que involucró una cohorte de 36.177 varones y 40.484 mujeres orientales de 45-75 años con un seguimiento de 11 años, se observó una asociación inversamente proporcional entre la ingesta de isoflavonas y el riesgo cáncer de pulmón en varones y mujeres que nunca habían sido fumadores, pero no se observó dicha asociación en sujetos fumadores o ex fumado$\mathrm{res}^{74}$. Otro estudio, realizado en 24.127 mujeres orientales de mediana edad con un seguimiento de más de 13 años, concluye que la genisteína sérica se relaciona con menor riesgo de cáncer de pulmón ${ }^{75}$.

Dos metaanálisis de estudios epidemiológicos (estudios prospectivos y estudios de casos y controles) concluyen que existe una asociación estadísticamente significativa entre la ingesta de flavonoides y la disminución del riesgo de cáncer $^{19,20}$. En uno de dichos metaanálisis se describe que la alta ingesta de flavonoides ( $20 \mathrm{mg} /$ día) se puede asociar, de manera estadísticamente significativa, con una reducción del $10 \%$ en el cáncer de pulmón ${ }^{20}$. Sin embargo, también hay estudios epidemiológicos que han hallado relaciones contradictorias, hecho que se debe tener en cuenta cuando se analiza la evidencia existente. Un estudio, publicado en 1998 y realizado en una cohorte española, concluye que no se observó asociación clínicamente significativa entre la ingesta de caempferol, quercetina, luteolina o total de flavonoides y el riesgo de padecer cáncer de pulmón ${ }^{23}$. Otro estudio prospectivo, que involucró una cohorte de 38.408 mujeres de mediana edad durante un periodo de seguimiento de más de 11 años, concluye que no se ha hallado una asociación estadísticamente significativa entre la ingesta de los cinco flavonoides más comunes o una selección de los alimentos ricos en flavonoides y la prevención de ningún tipo de cáncer (incluido el de pulmón) ${ }^{27}$. Una revisión sistemática publicada en 2012, que incluyó un metaanálisis, una revisión sistemática, 11 estudios prospectivos y 5 estudios de casos y controles en su análisis, concluye que el efecto protector contra el cáncer de pulmón del consumo de flavonoides fue pequeño y que no todos los estudios lo confirman ${ }^{24}$. Así, algunos autores apuntan la necesidad de realizar más estudios, concluyen que la evidencia actual es escasa y advierten de la posibilidad de que los diseños de algunos estudios epidemiológicos sean erróneos ${ }^{27}$.

\section{Conclusiones}

Pese a que estudios realizados en modelos celulares y animales muestran la capacidad potencial de los flavonoides para actuar contra diferentes tipos de cáncer, en especial el de pulmón, y que los posibles mecanismos de acción para esa actividad de los flavonoides actualmente están mejor estudiados, la presencia de datos contradictorios en los estudios epidemiológicos y la falta de estudios clínicos en humanos impiden realizar recomendaciones dietéticas basadas en la evidencia científica. Sin embargo, a la espera de nuevos y mejores estudios, los autores del presente artículo consideran oportuno seguir los consejos de las guías de alimentación saludable, que promocionan el consumo de alimentos ricos en flavonoides como, por ejemplo, la promoción de cinco o más raciones de fruta y verdura al día. Asimismo, cabe esperar que una dieta o un estilo de vida mediterráneo - que implica seguir una dieta rica en frutas y hortalizas, cereales integrales y legumbres y, por lo tanto, rica en alimentos que contienen gran cantidad de flavonoides- y evitar el consumo de tabaco y realizar actividad física podrían ayudar en la disminución del riesgo o incluso en la prevención de muchas enfermedades crónicas, incluido el cáncer de pulmón. La promoción de la investigación de campo ofrecerá, en un futuro, la oportunidad de establecer recomendaciones específicas de ingesta de flavonoides a través de la dieta.

\section{Conflicto de intereses}

Los autores declaran que no tienen ninguna relación personal o financiera con otras personas u organizaciones que puedan influir de manera inapropiada en su trabajo. 


\section{Bibliografía}

1. Willett WC, Sacks F, Trichopoulou A, Drescher G, Ferro-Luzzi A, Helsing E, et al. Mediterranean diet pyramid: a cultural model for healthy eating. Am J Clin Nutr. 1995;61(6 Suppl): S1402-6.

2. Bielsaki HK, Grimm P. En: Nutrición, texto y atlas. $1 .^{\mathrm{a}}$ ed. Madrid: Panamericana; 2007.

3. Willett W. Nutrition and cancer: the search continues. Nutr Cancer. 2008;60:557-9.

4. Aune D, Lau R, Chan DS, Vieira R, Greenwood DC, Kampman E, et al. Nonlinear reduction in risk for colorectal cancer by fruit and vegetable intake based on meta-analysis of prospective studies. Gastroenterology. 2011;141:106-18.

5. Lunet N, Valbuena C, Vieira AL, Lopes C, David L, Carneiro F, et al. Fruit and vegetable consumption and gastric cancer by location and histological type: case-control and meta-analysis. Eur J Cancer Prev. 2007;16:312-27.

6. Pavia M, Pileggi C, Nobile CG, Angelillo IF. Association between fruit and vegetable consumption and oral cancer: a metaanalysis of observational studies. Am J Clin Nutr. 2006;83:112634.

7. Yang WS, Va P, Wong MY, Zhang HL, Xiang YB. Soy intake is associated with lower lung cancer risk: results from a metaanalysis of epidemiologic studies. Am J Clin Nutr. 2011;94:157583.

8. Hwang YW, Kim SY, Jee SH, Kim YN, Nam CM. Soy food consumption and risk of prostate cancer: a meta-analysis of observational studies. Nutr Cancer. 2009;61:598-606.

9. Butler $L M$, Wu AH. Green and black tea in relation to gynecologic cancers. Mol Nutr Food Res. 2011;55:931-40.

10. Mehta RG, Murillo G, Naithani R, Peng X. Cancer chemoprevention by natural products: how far have we come? Pharm Res. 2010;27:950-61.

11. Thomasset SC, Berry DP, Garcea G, Marczylo T, Steward WP, Gescher AJ. Dietary polyphenolic phytochemicals - promising cancer chemopreventive agents in humans? A review of their clinical properties. Int J Cancer. 2007;120:451-8.

12. Mikkelsen TB, Osler M, Orozova-Bekkevold I, Knudsen VK, Olsen SF. Association between fruit and vegetable consumption and birth weight: a prospective study among 43,585 Danish women. Scand J Public Health. 2006;34:616-22.

13. Malinska D, Kiersztan A. Flavonoids -characteristics and significance for therapy. Postepy Biochem. 2004;50:182-96.

14. Meiyanto E, Hermawan A, Anindyajati A. Natural products for cancer-targeted therapy: citrus flavonoids as potent chemopreventive agents. Asian Pac J Cancer Prev. 2012;13:42736.

15. Murakami A, Ashida H, Terao J. Multitargeted cancer prevention by quercetin. Cancer Lett. 2008;269:315-25.

16. Ekstrom AM, Serafini M, Nyren O, Wolk A, Bosetti C, Bellocco R. Dietary quercetin intake and risk of gastric cancer: results from a population-based study in Sweden. Ann Oncol. 2011;22:438-43.

17. Yao H, Xu W, Shi X, Zhang Z. Dietary flavonoids as cancer prevention agents. J Environ Sci Health C Environ Carcinog Ecotoxicol Rev. 2011;29:1-31.

18. Birt DF, Hendrich S, Wang W. Dietary agents in cancer prevention: flavonoids and isoflavonoids. Pharmacol Ther. 2001;90:157-77.

19. Neuhouser ML. Dietary flavonoids and cancer risk: evidence from human population studies. Nutr Cancer. 2004;50:1-7.

20. Tang NP, Zhou B, Wang B, Yu RB, Ma J. Flavonoids intake and risk of lung cancer: a meta-analysis. Jpn J Clin Oncol. 2009;39:352-9.

21. Arts IC. A review of the epidemiological evidence on tea, flavonoids, and lung cancer. J Nutr. 2008;138:1561-6.
22. Le Marchand L, Murphy SP, Hankin JH, Wilkens LR, Kolonel LN. Intake of flavonoids and lung cancer. J Natl Cancer Inst. 2000;92:154-60.

23. Garcia-Closas R, Agudo A, Gonzalez CA, Riboli E. Intake of specific carotenoids and flavonoids and the risk of lung cancer in women in Barcelona, Spain. Nutr Cancer. 1998;32:154-8.

24. Garcia-Tirado J, Rieger-Reyes C, Saz-Peiro P. Effect of flavonoids in the prevention of lung cancer: systematic review. Med Clin (Barc). 2012;139:1-6.

25. Knekt P, Jarvinen R, Seppanen R, Hellovaara M, Teppo L, Pukkala $\mathrm{E}$, et al. Dietary flavonoids and the risk of lung cancer and other malignant neoplasms. Am J Epidemiol. 1997;146:223-30.

26. Hirvonen T, Virtamo J, Korhonen P, Albanes D, Pietinen P. Flavonol and flavone intake and the risk of cancer in male smokers (Finland). Cancer Causes Control. 2001;12:789-96.

27. Wang L, Lee IM, Zhang SM, Blumberg JB, Buring JE, Sesso HD. Dietary intake of selected flavonols, flavones, and flavonoidrich foods and risk of cancer in middle-aged and older women. Am J Clin Nutr. 2009;89:905-12.

28. Jemal A, Siegel R, Ward E, Hao Y, Xu J, Thun MJ. Cancer statistics, 2009. CA Cancer J Clin. 2009;59:225-49.

29. Ferlay J, Parkin DM, Steliarova-Foucher E. Estimates of cancer incidence and mortality in Europe in 2008. Eur J Cancer. 2010;46:765-81.

30. Egert S, Rimbach $\mathrm{G}$. Which sources of flavonoids: complex diets or dietary supplements? Adv Nutr. 2011;2:8-14.

31. Williamson G, Sies H, Heber D, Keen CL, Macdonald IA, ActisGoretta L, et al. Functional foods for health promotion: stateof-the-science on dietary flavonoids. Extended abstracts from the 12th Annual Conference on Functional Foods for Health Promotion, April 2009. Nutr Rev. 2009;67:736-43.

32. Mlodzinska E. Survey of plant pigments: molecular and environmental determinants of plant colors. Act Biol Crac Ser Bot. 2009;51:7-16.

33. Mo Y, Nagel C, Taylor LP. Biochemical complementation of chalcone synthase mutants defines a role for flavonols in functional pollen. Proc Natl Acad Sci U S A. 1992;89:7213-7.

34. Wiseman BR, Snook M, Widstrom NW. Feeding responses of the corn ear worm larvae (Lepidoptera: Noctuidae) on corn silks of varying flavone content. J Econ Entomol. 1996;89:1040-4.

35. Dixon RA, Steele CL. Flavonoids and isoflavonoids $-\mathrm{a}$ gold mine for metabolic engineering. Trends Plant Sci. 1999;4:394400.

36. Grotewold E. The science of flavonoids. New York: Springer; 2006.

37. Di Carlo G, Mascolo N, Izzo AA, Capasso F. Flavonoids: old and new aspects of a class of natural therapeutic drugs. Life Sci. 1999;65:337-53.

38. Bhagwat S, Haytowitz DB, Holden JM. USDA Database for the flavonoid content of selected foods. Baltimore: US Department of Agriculture; 2011.

39. Chabot GG, Touil YS, Pham MH, Dauzonne D. Flavonoids in cancer prevention and therapy: chemistry, pharmacology, mechanisms of action, and perspectives for cancer drug discovery. En: Alaoui-Jamali $M$, editor. Alternative and complementary therapies for cancer. New York: Springer; 2010.

40. Grassi D, Desideri G, Croce G, Tiberti S, Aggio A, Ferri C. Flavonoids, vascular function and cardiovascular protection. Curr Pharm Des. 2009;15:1072-84.

41. Huang Y, Tsang SY, Yao X, Chen ZY. Biological properties of baicalein in cardiovascular system. Curr Drug Targets Cardiovasc Haematol Disord. 2005;5:177-84.

42. Andres A, Donovan SM, Kuhlenschmidt MS. Soy isoflavones and virus infections. J Nutr Biochem. 2009;20:563-9.

43. Mota KS, Dias GE, Pinto ME, Luiz-Ferreira A, Souza-Brito AR, Hiruma-Lima CA, et al. Flavonoids with gastroprotective activity. Molecules. 2009;14:979-1012. 
44. Cheong H, Ryu SY, Oak MH, Cheon SH, Yoo GS, Kim KM. Studies of structure activity relationship of flavonoids for the antiallergic actions. Arch Pharm Res. 1998;21:478-80.

45. Stoclet JC, Chataigneau T, Ndiaye M, Oak MH, El Bedoui J, Chataigneau $M$, et al. Vascular protection by dietary polyphenols. Eur J Pharmacol. 2004;500:299-313.

46. Gilani AH, Janbaz KH, Shah BH. Quercetin exhibits hepatoprotective activity in rats. Biochem Soc Trans. 1997;25: S619.

47. Majumdar S, Srirangam R. Potential of the bioflavonoids in the prevention/treatment of ocular disorders. J Pharm Pharmacol. 2010;62:951-65.

48. Spencer JP. The impact of fruit flavonoids on memory and cognition. Br J Nutr. 2010;104:40-7.

49. Chahar MK, Sharma N, Dobhal MP, Joshi YC. Flavonoids: A versatile source of anticancer drugs. Pharmacogn Rev. 2011;5:1-12.

50. Liao YC, Shih YW, Chao CH, Lee XY, Chiang TA. Involvement of the ERK signaling pathway in fisetin reduces invasion and migration in the human lung cancer cell line A549. J Agric Food Chem. 2009;57:8933-41.

51. Hsiao YC, Kuo WH, Chen PN, Chang HR, Lin TH, Yang WE, et al. Flavanone and 2'-OH flavanone inhibit metastasis of lung cancer cells via down-regulation of proteinases activities and MAPK pathway. Chem Biol Interact. 2007;167:193-206.

52. Cai X, Ye T, Liu C, Lu W, Lu M, Zhang J, et al. Luteolin induced G2 phase cell cycle arrest and apoptosis on non-small cell lung cancer cells. Toxicol In Vitro. 2011;25:1385-91.

53. Moon YJ, Wang X, Morris ME. Dietary flavonoids: effects on xenobiotic and carcinogen metabolism. Toxicol In Vitro. 2006;20:187-210.

54. Nguyen TT, Tran E, Ong CK, Lee SK, Do PT, Huynh TT, et al. Kaempferol-induced growth inhibition and apoptosis in A549 lung cancer cells is mediated by activation of MEK-MAPK. J Cell Physiol. 2003;197:110-21.

55. Nguyen TT, Tran E, Nguyen TH, Do PT, Huynh TH, Huynh H. The role of activated MEK-ERK pathway in quercetin-induced growth inhibition and apoptosis in A549 lung cancer cells. Carcinogenesis. 2004;25:647-59.

56. Kuo PC, Liu HF, Chao Jl. Survivin and p53 modulate quercetininduced cell growth inhibition and apoptosis in human lung carcinoma cells. J Biol Chem. 2004;279:55875-85.

57. Kosmider B, Wojcik I, Osiecka R, Bartkowiak J, Zyner E, Ochocki $\mathrm{J}$, et al. Enhanced P53 and BAX gene expression and apoptosis in A549 cells by cis-Pt(II) complex of 3-aminoflavone in comparison with cis-DDP. Invest New Drugs. 2005;23:287-97.

58. Schwarz D, Kisselev P, Roots I. CYP1A1 genotype-selective inhibition of benzo[a]pyrene activation by quercetin. Eur $\mathrm{J}$ Cancer. 2005;41:151-8.

59. Chen W, Wang X, Zhuang J, Zhang L, Lin Y. Induction of death receptor 5 and suppression of survivin contribute to sensitization of TRAIL-induced cytotoxicity by quercetin in non-small cell lung cancer cells. Carcinogenesis. 2007;28:2114-21.

60. Leung HW, Lin CJ, Hour MJ, Yang WH, Wang MY, Lee HZ. Kaempferol induces apoptosis in human lung non-small carcinoma cells accompanied by an induction of antioxidant enzymes. Food Chem Toxicol. 2007;45:2005-13.

61. Chen LG, Hung LY, Tsai KW, Pan YS, Tsai YD, Li YZ, et al. Wogonin, a bioactive flavonoid in herbal tea, inhibits inflammatory cyclooxygenase-2 gene expression in human lung epithelial cancer cells. Mol Nutr Food Res. 2008;52:1349-57.

62. Lee HJ, Lee EO, Ko SG, Bae HS, Kim CH, Ahn KS, et al. Mitochondria-cytochrome C-caspase-9 cascade mediates isorhamnetin-induced apoptosis. Cancer Lett. 2008;270:342-53.

63. Khan N, Mukhtar H. Cancer and metastasis: prevention and treatment by green tea. Cancer Metastasis Rev. 2010;29:435-45.

64. Du G, Han G, Zhang S, Lin H, Wu X, Wang M, et al. Baicalin suppresses lung carcinoma and lung metastasis by SOD mimic and HIF-1alpha inhibition. Eur J Pharmacol. 2010;630:121-30.

65. Hung JY, Hsu YL, Ko YC, Tsai YM, Yang CJ, Huang MS, et al. Didymin, a dietary flavonoid glycoside from citrus fruits, induces Fas-mediated apoptotic pathway in human non-smallcell lung cancer cells in vitro and in vivo. Lung Cancer. 2010; 68:366-74.

66. Anso E, Zuazo A, Irigoyen M, Urdaci MC, Rouzaut A, MartinezIrujo JJ. Flavonoids inhibit hypoxia-induced vascular endothelial growth factor expression by a HIF-1 independent mechanism. Biochem Pharmacol. 2010;79:1600-9.

67. Hsu HS, Lin JH, Huang WC, Hsu TW, Su K, Chiou SH, et al. Chemoresistance of lung cancer stemlike cells depends on activation of Hsp27. Cancer. 2011;117:1516-28.

68. Ravichandran N, Suresh G, Ramesh B, Siva GV. Fisetin, a novel flavonol attenuates benzo(a)pyrene-induced lung carcinogenesis in Swiss albino mice. Food Chem Toxicol. 2011;49: 1141-7.

69. Hasani A, Leighl N. Classification and toxicities of vascular disrupting agents. Clin Lung Cancer. 2011;12:18-25.

70. Jin CY, Park C, Hwang HJ, Kim GY, Choi BT, Kim WJ, et al. Naringenin up-regulates the expression of death receptor 5 and enhances TRAIL-induced apoptosis in human lung cancer A549 cells. Mol Nutr Food Res. 2011;55:300-9.

71. Lopez-Lazaro M. Flavonoids as anticancer agents: structureactivity relationship study. Curr Med Chem Anticancer Agents. 2002;2:691-714.

72. Cutler GJ, Nettleton JA, Ross JA, Harnack LJ, Jacobs DR Jr, Scrafford CG, et al. Dietary flavonoid intake and risk of cancer in postmenopausal women: the lowa Women's Health Study. Int J Cancer. 2008;123:664-71.

73. Mursu J, Nurmi T, Tuomainen TP, Salonen JT, Pukkala E, Voutilainen S. Intake of flavonoids and risk of cancer in Finnish men: The Kuopio Ischaemic Heart Disease Risk Factor Study. Int J Cancer. 2008;123:660-3.

74. Shimazu T, Inoue M, Sasazuki S, Iwasaki M, Sawada N, Yamaji T, et al. Isoflavone intake and risk of lung cancer: a prospective cohort study in Japan. Am J Clin Nutr. 2010;91:722-8.

75. Shimazu T, Inoue M, Sasazuki S, Iwasaki M, Sawada N, Yamaji T, et al. Plasma isoflavones and the risk of lung cancer in women: a nested case-control study in Japan. Cancer Epidemiol Biomarkers Prev. 2011;20:419-27. 